\title{
Rubinstein-Taybi syndrome
}

INSERM

\section{Source}

INSERM. (1999). Orphanet: an online rare disease and orphan drug data base. Rubinstein-

Taybisyndrome. ORPHA:783

Rubinstein-T aybi syndrome is a rare malformation syndrome characterized by congenital anomalies (microcephaly, specific facial characteristics, broad thumbs and halluces and postnatal growth retardation), short stature, intellectual disability and behavioural characteristics. 